\title{
Functional and T Cell Receptor Repertoire Analyses of Peripheral Blood and Infrapatellar Fat Pad T Cells in Knee Osteoarthritis
}

\author{
Thitiya Sae-jung, Panjana Sengprasert, Jirun Apinun, Srihatach Ngarmukos, \\ Pongsak Yuktanandana, Aree Tanavalee, and Rangsima Reantragoon
}

\begin{abstract}
Objective. Osteoarthritis (OA) is a condition that features inflammation and immune responses of innate and adaptive immunity. The role of T cells in knee OA pathogenesis is still unclear. Our aim was to characterize $\mathrm{T}$ cell functions and their clonality in patients with knee OA in peripheral blood (PB) and infrapatellar fat pads (IPFP).

Methods. We isolated T cells from PB and IPFP of patients with knee OA and PB of healthy individuals and determined soluble mediators produced from these cells. In addition, we performed a clonal analysis of activated CD8+ T cells and compared the T cell receptor $\beta$-variable gene chain (TRBV) usages between T cells in PB and IPFP of patients with knee OA.

Results. Our results suggest that in patients with knee OA, circulating $\mathrm{T}$ cells possess a more "cytotoxic" profile or rather impaired cytokine production, but the knee microenvironment allows for these T cells to produce proinflammatory cytokines [interleukin (IL)-1 $\beta$, IL-6, tumor necrosis factor], IL-17, and interferon- $\gamma$ within IPFP. Activated CD8+ IPFP T cells carry different repertoire distribution from those present in PB of patients with knee OA. Shared TRBV usage of activated CD8+ IPFP $\mathrm{T}$ cells among the 3 patients with knee OA was also observed.

Conclusion. Our study describes the nature of T cells in knee OA that may be due to "unhealthy" aging or other factors that drive healthy aging $\mathrm{T}$ cells into a state of imbalance, thus contributing to the pathogenesis of knee OA. (First Release October 15 2018; J Rheumatol 2019;46:309-17; doi:10.3899/ jrheum.170775)
\end{abstract}

Key Indexing Terms:

OSTEOARTHRITIS

T CELLS

INFLAMMATION

\section{CYTOTOXICITY}

INFRAPATELLAR FAT PAD T CELL RECEPTOR REPERTOIRE

From the Medical Microbiology Interdisciplinary Program, Graduate

School, Chulalongkorn University; Immunology Division, Department of

Microbiology, Faculty of Medicine, Chulalongkorn University:

Department of Orthopedics, Faculty of Medicine, Chulalongkorn

University; Center of Excellence in Immunology and Immune-mediated

Diseases, Faculty of Medicine, Chulalongkorn University, Bangkok, Thailand.

This project was supported by Faculty of Medicine Ratchadapiseksompot Grant, Grant no. RA58/054 and the Thai Research Fund, Grant no. MRG6080122. TS is an MS student supported by the Chulalongkorn University Graduate Scholarship to Commemorate the 72nd Anniversary of His Majesty King Bhumibol Adulyadej.

T. Sae-jung, BS, Medical Microbiology Interdisciplinary Program, Graduate School, Chulalongkorn University; P. Sengprasert, MS, Immunology Division, Department of Microbiology, Faculty of Medicine, Chulalongkorn University; J. Apinun, MD, Department of Orthopedics, Faculty of Medicine, Chulalongkorn University; S. Ngarmukos, MD, Department of Orthopedics, Faculty of Medicine, Chulalongkorn University; P. Yuktanandana, MD, Associate Professor of Orthopedics, Department of Orthopedics, Faculty of Medicine, Chulalongkorn University; A. Tanavalee, MD, Professor of Orthopedics, Department of Orthopedics, Faculty of Medicine, Chulalongkorn University;

R. Reantragoon, $M D$, PhD, Lecturer in Immunology, Immunology

Division, Department of Microbiology, Faculty of Medicine, and Center of

Excellence in Immunology and Immune-mediated Diseases, Faculty of

Medicine, Chulalongkorn University.

Address correspondence to Dr. R. Reantragoon, Faculty of Medicine, Chulalongkorn University, Microbiology, 1873 Rama 4 Road, Bangkok 10330, Thailand.E-mail: rangsima.reantragoon@gmail.com, Rangsima.R@chula.ac.th

Accepted for publication July 19, 2018.
Osteoarthritis (OA) is a debilitating disease featured by periarticular tissue dysfunction and inflammation of surrounding tissues, e.g., fat pads ${ }^{1,2}$. It was originally described as a degenerative condition ${ }^{1,3}$ from biomechanical loading of the joints, but current evidence suggests immune responses may also be involved in OA development ${ }^{4,5,6}$.

Immune responses related to knee OA include both innate and adaptive immune compartments $7,8,9,10$. Low-grade inflammation is well described in knee OA, with presence of soluble proteins and inflammatory mediators in synovial fluids ${ }^{5,11,12}$. Infiltration of inflammatory immune cells within the infrapatellar fat pad (IPFP) and synovium are also key findings in knee OA pathology $13,14,15$. The IPFP (or Hoffa's pad) is an intraarticular and intracapsular adipose tissue that serves as a local producer of adipocytokines under the influence of local stimuli ${ }^{13,16,17}$. It has been proposed that IPFP may serve for both biomechanical (pressure absorption) and biochemical (inflammatory mediator secretion) purposes in maintaining the integrity of the knee joint ${ }^{18}$. Tissue composition of IPFP includes adipocytes, immune cells, nerve tissues, and connective tissue ${ }^{13}$ and resembles that of visceral adipose tissue $^{13}$. IPFP volume may affect knee OA development, but studies are still being debated $19,20,21,22,23$.

Personal non-commercial use only. The Journal of Rheumatology Copyright @ 2019 . All rights reserved. 
Regardless, the IPFP is a site where inflammatory cells can accumulate, including macrophages, T cells, B cells, mast cells, and others ${ }^{24,25}$. In our study we mainly focus on IPFP T cells.

T cells are the second most abundant immune cells within IPFP $^{25}$. There are many studies investigating the role of $\mathrm{T}$ cells in the pathogenesis of osteoarthritis ${ }^{10}$, but fewer studies of IPFP-infiltrating T cells ${ }^{24,25}$. Mice lacking CD4+ or $\mathrm{CD} 8+\mathrm{T}$ cells show a decrease in severity of knee OA, suggesting their role in driving knee OA pathology 26,27 . However, the mechanisms are still undefined. Thus, it was in our interest to investigate T cells from IPFP and peripheral blood of patients with knee OA. In this study, we characterized $\mathrm{T}$ cell function by determining the levels of cytokine and cytotoxic molecule production from IPFP T cells and peripheral blood $\mathrm{T}$ cells of patients with knee OA. In addition, we analyzed the $\mathrm{T}$ cell receptor (TCR) repertoire of IPFP and peripheral blood $\mathrm{T}$ cells of these patients.

\section{MATERIALS AND METHODS}

Patient recruitment and tissue sample collection. Patients with knee OA and healthy individuals were recruited from King Chulalongkorn Memorial Hospital. Peripheral blood and IPFP were obtained from patients with knee OA undergoing total knee arthroplasty, while peripheral blood came from healthy individuals. For ethical reasons, we were not able to obtain healthy IPFP from living donors, and access to postmortem IPFP was limited. Procedures were performed in accordance with the ethical standards and with approval from the committee on human experimentation (Institutional Review Board) at the Faculty of Medicine, Chulalongkorn University, Bangkok, Thailand (IRB no. 574/57) and with the Helsinki Declaration of 1975, as revised in 2000. All samples were obtained with the patient's/ individual's signed consent. Tissue samples obtained were processed immediately.

Peripheral blood mononuclear cell isolation. Whole blood was layered on to Ficoll-Paque (GE Healthcare) and centrifuged at $2000 \mathrm{rpm}$ for $20 \mathrm{~min}$ at room temperature (no deceleration force). Cells were washed twice with RPMI 1640 (Life Technologies) supplemented with 10\% fetal calf serum (FCS; Life Technologies) and cryopreserved in $90 \% \mathrm{FCS} / 10 \%$ dimethyl sulfoxide (DMSO; Amresco) until experiments were performed.

IPFP T cell isolation. Fat pads were washed with phosphate buffer saline (PBS), cut into 2-3 mm pieces, and digested with $3 \mu \mathrm{g} / \mathrm{ml}$ collagenase type IV (Worthington), $0.1 \mu \mathrm{g} / \mathrm{ml}$ DNase I (Worthington), and 5\% FCS in PBS with a shaking force of $200 \mathrm{rpm}, 37^{\circ} \mathrm{C}$ for $90 \mathrm{~min}$. The supernatant was filtered through a $40-\mu \mathrm{m}$ filter and washed with RPMI 1640 (Life Technologies) supplemented with $10 \%$ FCS and cryopreserved in 90\%FCS/10\% DMSO (Amresco).

Ex vivo $T$ cell analysis. For cytokine detection, $10^{5}$ mononuclear cells were cultured in the presence of anti-CD3/anti-CD28 beads (Thermofisher Scientific) and brefeldin A (BFA) added (final $20 \mu \mathrm{g} / \mathrm{ml}$ ) and incubated at $37^{\circ} \mathrm{C}$ for $16-18 \mathrm{~h}$. Cells were labeled with anti-CD3-PE-Cy7 (clone UCHT1), anti-CD4-PE-Cy5 (clone RPA-T4), and anti-CD8-PerCp/Cy5.5 (clone SK1) antibodies at $4^{\circ} \mathrm{C}$ for $30 \mathrm{~min}$, then fixed with $1 \%$ formaldehyde in PBS at $4^{\circ} \mathrm{C}$ for $30 \mathrm{~min}$ and further labeled with anti-interleukin (IL)-17-PE (clone SCPL1362), anti-IL1- $\beta$-FITC (clone H1b-98), anti-IL-6-APC (clone MQ2-13A5), anti-interferon (IFN)- $\gamma$-APC-Cy7 (clone 4S.B3), and anti-tumor necrosis factor (TNF)-AlexaFluor 700 (clone MAb11) antibodies in $0.3 \%$ saponin (Sigma-Aldrich).

For cytotoxic molecule detection, $10^{5}$ cells were initially labeled with anti-CD107a-PE-Cy7 (clone H4A3) antibody and stimulated with anti-CD3/anti-CD28 beads (Thermofisher Scientific) for $1 \mathrm{~h}$, then BFA (final $20 \mu \mathrm{g} / \mathrm{ml}$ ) and monensin (final $20 \mu \mathrm{g} / \mathrm{ml}$ ) were added and incubated at $37^{\circ} \mathrm{C}$ for 16-18 h. Cells were washed and labeled with anti-CD3-APC (clone UCHT1), anti-CD4-APC-Cy7 (clone RPA-T4), and anti-CD8-AF700 (clone SK1) antibodies at $4^{\circ} \mathrm{C}$ for $30 \mathrm{~min}$, then fixed with $1 \%$ formaldehyde in PBS and labeled with antigranzyme B-FITC (clone GB11), anti-perforin-PerCp/Cy5.5 (clone SG9), and anti-granulysin-PE (clone DH2) antibodies in $0.3 \%$ saponin (Sigma-Aldrich). All antibodies were purchased from Biolegend. Cells were then acquired on the BD LSRII flow cytometer. Analysis was performed using the Flowjo software (Treestar; Supplementary Figures $1-3$, available with the online version of this article).

$T C R$ repertoire determination. Cells were labeled with anti-CD3-APC (clone UCHT1), anti-CD8-AF700 (clone SK1), and anti-CD69-PE (clone FN50; Biolegend) antibodies. CD3+CD8+CD69+ cells were single cell-sorted into 96-V well PCR plates using the BD FACS Aria II-cell sorter. cDNA was synthesized using SuperScript VILO cDNA Synthesis Kit (Invitrogen) in $2.5 \mu 1$ reaction mixes as the manufacturer's description. Products were amplified by multiplex nested PCR for TCR transcripts. Primers were used as previously described ${ }^{28}$. In the first round, $2.5 \mu 1$ of cDNA was added to $2.5 \mu 1$ of $10 \times$ PCR buffer, $1.5 \mathrm{mM} \mathrm{MgCl}{ }^{2}, 0.2 \mathrm{mM}$ deoxynucleotide triphosphate, $0.75 \mathrm{U}$ of Taq DNA polymerase, and $2.5 \mathrm{pmol}$ each of the external sense (TRBV and TRAV) and external antisense (TRBC and TRAC) primers $^{28}$. Then, $5 \mu$ l of PCR products were used as templates for subsequent rounds replacing the primers with internal sense TRAV primers and internal antisense TRAC primers; or internal sense TRBV primers and internal antisense TRBC primers ${ }^{28}$. PCR reaction mixes were incubated at $95^{\circ} \mathrm{C}$ for $2 \mathrm{~min}$, following 35 cycles of $95^{\circ} \mathrm{C}$ for $20 \mathrm{~s}, 52^{\circ} \mathrm{C}$ for $20 \mathrm{~s}, 72^{\circ} \mathrm{C}$ for $45 \mathrm{~s}$, and 1 cycle of $72^{\circ} \mathrm{C}$ for $7 \mathrm{~min}$. DNA products were detected by $2 \%$ agarose gel electrophoresis and amplified using internal antisense TRAC or TRBC primer after treatment with Illustra ExoProStar 1-Step Kit (GE Healthcare). Data were analyzed using the ImMunoGeneTics database.

Statistical analysis. All data were analyzed using the Statistical Package for Social Sciences (SPSS 15.0, SPSS Inc.) or Graph Pad InStat version 5.0 software. The results were presented as mean \pm SD. Independent sample t tests was used to compare the value between 2 groups (between healthy control and patients with knee OA; and peripheral blood and IPFP of patients with knee OA). Statistical significance was defined as $\mathrm{p} \leq 0.05, \mathrm{p} \leq 0.01$, or $\mathrm{p} \leq 0.001$

\section{RESULTS}

Demographic characteristics of studied populations. Patients with knee OA $(\mathrm{n}=31)$ and healthy individuals $(\mathrm{n}=11)$ were included in the study (Table 1). Demographic characteristics showed mean values ( \pm SD values) of age, sex distribution, weight, height, and body mass index (BMI; Table 1). The mean age, weight, and BMI of patients with knee OA was higher than those of healthy individuals, with both populations having comparable mean height (Table 1).

Peripheral blood $T$ cell cytokine and cytotoxic mediator profiling of patients with knee OA. Inflammation in knee OA is characterized by an imbalance between catabolic and anabolic cytokines ${ }^{29}$. Among the catabolic cytokines, IL-1 $\beta$, IL-6, and TNF are well-described cytokines related to knee $\mathrm{OA}^{30,31}$ and can be secreted from $\mathrm{T}$ cells ${ }^{32,33}$. In addition, pathogenic IL-17-producing Th cells have been shown to be pivotal in the pathogenesis of rheumatoid arthritis ${ }^{34}$. IFN- $\gamma$ is a $\mathrm{T}$ cell signature cytokine secreted in response to antigenic stimulation, in which autoantigens have been proposed in knee $\mathrm{OA}^{35}$. For these reasons, we evaluated for $\mathrm{T}$ cell production of IL-1 $\beta$, IL-6, TNF, IL-17, and IFN- $\gamma$ from patients with knee OA and healthy individuals. First, peripheral blood $\mathrm{T}$ cells were isolated and evaluated for their 
Table 1. Demographic characteristics between healthy individuals and osteoarthritic patients.

\begin{tabular}{lccc}
\hline Characteristics & Healthy, $\mathrm{n}=11$ & Patients with OA, $\mathrm{n}=31$ & $\mathrm{p}$ \\
\hline Age, $\mathrm{yrs}$ & $55.55(7.31)$ & $68.88(7.91)$ & $<0.0001$ \\
Sex, $\mathrm{n}(\%)$ & & & \\
$\quad$ Male & $6.00(55)$ & $6.00(19)$ & \\
$\quad$ Female & $5.00(46)$ & $25.00(81)$ & 0.502 \\
Weight, $\mathrm{kg}$ & $59.23(9.67)$ & $61.81(11.26)$ & 0.008 \\
Height, $\mathrm{m}$ & $1.59(6.27)$ & $1.54(0.06)$ & 0.050 \\
BMI, $\mathrm{kg} / \mathrm{m}^{2}$ & $23.10(2.84)$ & $26.35(4.76)$ & \\
\hline
\end{tabular}

Data are mean (SD) except where indicated. OA: osteoarthritis; BMI: body mass index.

A

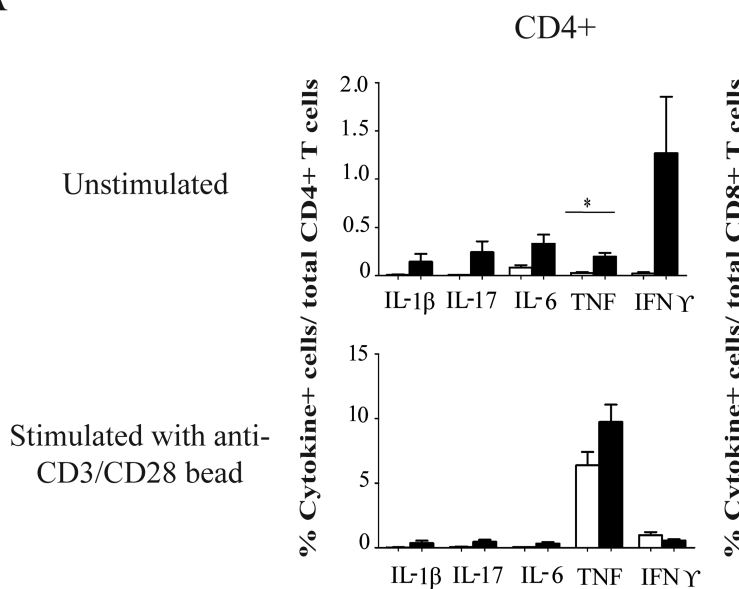

$\mathrm{B}$

$\mathrm{CD} 107 \mathrm{a}+\mathrm{CD} 4+$

$\mathrm{CD} 107 \mathrm{a}+\mathrm{CD} 8+$
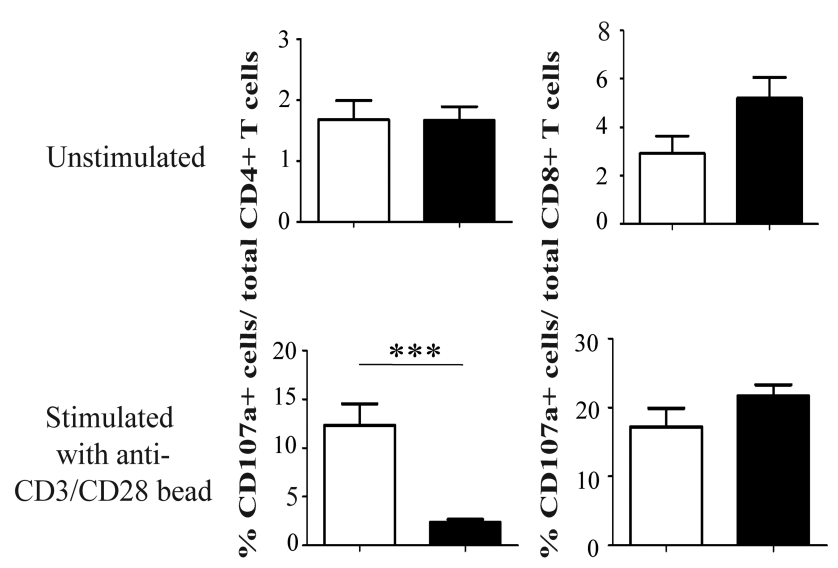

cytokine production ex vivo (unstimulated). We observed a significant increase in TNF production from CD4+ T cells and significant decreases in IL- $6, \mathrm{TNF}$, and IFN- $\gamma$ production from CD8+ T cells (Figure 1A). When these cells were stimulated with anti-CD3/anti-CD28 beads, cytokine level production between patients with knee OA and healthy individuals was mostly comparable, with only IL-6-producing and IFN- $\gamma$-producing CD8+ T cells in patients with knee OA showing significantly lower levels of cytokine production than those in healthy individuals (Figure 1A). These findings may suggest an impaired function in IL-6 and IFN- $\gamma$ production from peripheral blood $\mathrm{T}$ cells in patients with knee OA compared to healthy individuals.

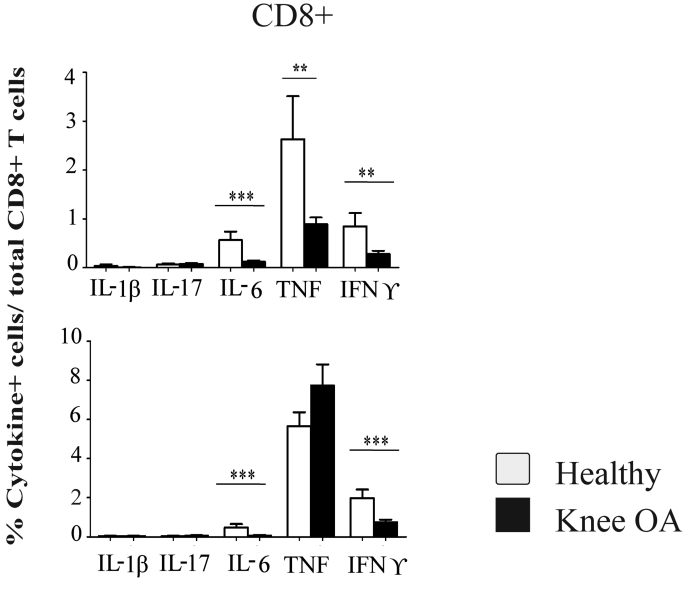

Figure 1. Peripheral blood T cell cytokine and cytotoxic molecule production of patients with knee OA and healthy individuals. Peripheral blood T cells from knee OA patients and healthy individuals were isolated and stimulated with anti-CD3/anti-CD28 beads. Intracellular cytokine staining and degranulation assay was performed to evaluate the levels of T cells producing the following: IL-1 $\beta$, IL-17, IL-6, TNF, IFN- $\gamma$, perforin, granzyme B, and granulysin. T cells were grouped based on their coreceptor expression of CD4 or CD8. In all settings, T cells were evaluated in 2 conditions: (1) ex vivo (unstimulated) and (2) stimulated with anti-CD3/anti-CD28 beads. Shown are mean values. Error bars represent SD values. Healthy individuals (white bars), $\mathrm{n}=11 ;$ patients with knee OA (black bars), $\mathrm{n}=31 . * \mathrm{p} \leq 0.05, * * \mathrm{p} \leq 0.01, * * * \mathrm{p} \leq 0.001$. A. Percentage of cytokine+ T cells from total CD4+ (or CD8+) T cells in unstimulated (top panel) and stimulated (bottom panel) conditions. B. Percentage of CD107a+CD4+ and CD107a+CD8+ T cells from total CD4+ and total CD8+ T cells, respectively, in unstimulated (top panel) and stimulated (bottom panel) conditions. C. Percentage of cytotoxic molecule production from total CD107a+CD4+ or CD107a+CD8+ T cells in unstimulated (top panel) and stimulated (bottom panel) conditions. Prf: perforin; GrB: granzyme B; GNLY: granulysin; IL: interleukin; TNF: tumor necrosis factor; OA: osteoarthritis; IFN: interferon.

Personal non-commercial use only. The Journal of Rheumatology Copyright $\odot$ 2019. All rights reserved. 
A feature of knee OA is cartilage destruction ${ }^{9}$. We hypothesized that chondrocytes may serve as target cells for T cellmediated cytotoxicity. Therefore, we evaluated production of cytotoxic molecules secreted from peripheral blood $\mathrm{T}$ cells of patients with knee OA. Degranulation levels of T cells were also determined, as represented by cell surface expression of CD107a. Degranulation levels of ex vivo CD4+ and CD8+ $\mathrm{T}$ cells in patients with knee OA and healthy individuals were comparable (Figure 1B). However, after anti-CD3/anti-CD28 bead stimulation, cell surface expression of CD107a of knee OA peripheral blood $\mathrm{CD} 4+\mathrm{T}$ cells were not upregulated to levels comparable to those in healthy individuals (Figure 1B). Thus, we further selected only degranulating cells (CD107a+ cells) to determine cytotoxic molecule production. Our results showed that ex vivo CD8+CD107a+ T cells expressed significantly higher levels of perforin production. After T cell stimulation with anti-CD3/anti-CD28 beads, perforin and granulysin production of peripheral blood CD4+ and CD8+ $\mathrm{T}$ cells from patients with knee OA were increased when compared to healthy individuals, with significant increases seen in CD107a+CD4+perforin+, CD107a+CD4+ granulysin+, and CD107a+CD8+granulysin+ T cells (Figure 1C). The inability of CD4+ T cells to express levels of cell surface CD107a comparable to healthy individuals along with increased abundance of perforin and granulysin production after stimulation suggests that peripheral blood CD4+ T cells in patients with knee OA may be constantly polarized to cytotoxic CD4+ T cells. However, CD8+ T cells from patients with knee OA still exhibited their ability to be activated by anti-CD3/anti-CD28 bead stimulation and also showed a cytotoxicity profile. Altogether, our results here suggest that peripheral blood $\mathrm{T}$ cells in patients with knee OA are biased toward a cytotoxicity profile.

A comparison of IPFP T cells and peripheral blood T cells in patients with knee OA. The IPFP is the most adjacent site of adipose tissue to the knee joint and is described as a site of inflammatory cell infiltration ${ }^{25}$. Thus, we further evaluated $\mathrm{T}$ cells isolated from IPFP for their cytokine and cytotoxic molecule production. Owing to the limited number of isolated cells, we determined only the levels of ex vivo (unstimulated) conditions. Our study also lacks healthy IPFP for direct comparison.

Our results show that both CD4+ and CD8+ IPFP T cells had significantly higher levels of IL-1 $\beta$, IL-6, and TNF production than did peripheral blood $\mathrm{T}$ cells (Figure 2A). In addition, we observed significantly higher levels of IFN- $\gamma$ production in CD8+ T cells $(\mathrm{p}=0.003)$ and significantly higher levels of IL-17 production in CD4+ T cells (Figure $2 \mathrm{~A})$. Cell surface expression of $\mathrm{CD} 107$ a revealed higher levels of degranulation of IPFP CD4+ T cells $(p<0.0001)$ than peripheral blood of patients with knee OA, but levels of $\mathrm{CD} 107 \mathrm{a}$ for $\mathrm{CD} 8+\mathrm{T}$ cells between the 2 tissues were comparable (Figure 2B). We then determined cytotoxic molecule production of IPFP T cells regardless of their cell surface
CD107a. IPFP T cells exhibited increased levels of cytotoxicity as shown with significant increases of perforin-secreting and granulysin-secreting CD4+ $\mathrm{T}$ cells and perforin producing and granzyme B-producing CD8+ T cells (Figure 2C). These findings reflect $\mathrm{T}$ cells as potential sources of cytokines (TNF, IL-6, IL-1 $\beta$, IL-17, and IFN- $\gamma$ ) and cytotoxic molecules (perforin, granzyme $\mathrm{B}$, and granulysin) within IPFP. However, the extent of their contribution to local inflammation within the knee joint requires further evaluation. We further assessed the ability of cytokine-producing $\mathrm{T}$ cells to produce multiple cytokines. Our results show that the majority of IPFP T cells secreted only 1 cytokine; IL-1 $\beta$ and IL-6 were the most dominant (Figure 2D). A very few dual-cytokine secreting T cells were observed (Figure 2D).

Clonal analysis of activated CD8+T cell usage in patients with knee OA. Knee OA is correlated with increased BMI values $^{36}$, but not directly with weight ${ }^{37,38}$. Increased body weight is linked to alterations of adipose tissue function and metabolic states described to be associated with OA prevalence ${ }^{39,40}$. Mice fed a high-fat diet, which leads to increased weight, had initial migration of $\mathrm{CD} 8+\mathrm{T}$ cells to adipose tissues, followed by other immune cells ${ }^{41}$. These adipose tissue $\mathrm{T}$ cells also display a restricted TCR repertoire ${ }^{42}$. Previously, we have shown that in patients with knee OA, circulating CD8+ T cells were more activated than CD4+ $\mathrm{T}$ cells, and increased frequency of IPFP CD8+ T cells was observed with higher radiographic grading of knee $\mathrm{OA}^{15}$. In addition, Ponchel, et al detected a correlation between increasing numbers of peripheral blood CD8+ T cells, but not $\mathrm{CD} 4+\mathrm{T}$ cells, with age in osteoarthritic patients ${ }^{43}$. Thus, we investigated the TCR repertoire of activated CD8+ T cells in the IPFP of patients with OA and compared them to peripheral blood $\mathrm{T}$ cell clones by performing a clonal analysis of $\beta$-chain usages of CD8+CD69+ T cells in both tissues from 3 patients. Clonal analysis revealed that activated CD8+ $\mathrm{T}$ cells in IPFP have different repertoire patterns of TRBV usage from the peripheral blood in each individual (Figure $3 \mathrm{~A})$. The repertoire profiling showed that each donor also contained different predominant TRBV usage between IPFP and peripheral blood (Figure 3A and 3B). Peripheral blood activated CD8+ T cells were dominated by TRBV 15 and 20-1; TRBV 28 and 4-1; and TRBV 6-4, 20-1, and 15; for donors 1, 2, and 3, respectively (Figure 3A; Table 2). IPFP activated CD8+ T cells were dominated by TRBV 15, 27, 29-1, 2, and 13; TRBV 15, 19, and 20-1; and TRBV 20-1, 4-1, 19, and 29-1 for donors 1, 2, and 3, respectively (Figure $3 \mathrm{~A}$, Table 2). Comparison of activated CD8+ T cell clones in peripheral blood and IPFP among the 3 patients (interdonor comparison) demonstrates that in peripheral blood, shared TRBV usage was seen in TRBV 6-4, 20-1, and 28 (Figure $4 \mathrm{~A})$, but these did not represent the shared TRBV usage present in IPFP. We observed that IPFP-activated CD8+ $\mathrm{T}$ cells between each patient with knee OA possessed overlapping TRBV usages that were present as predominant

Personal non-commercial use only. The Journal of Rheumatology Copyright $\odot$ 2019. All rights reserved 
D

B
Knee OA PBMC

Knee OA IPFP
A

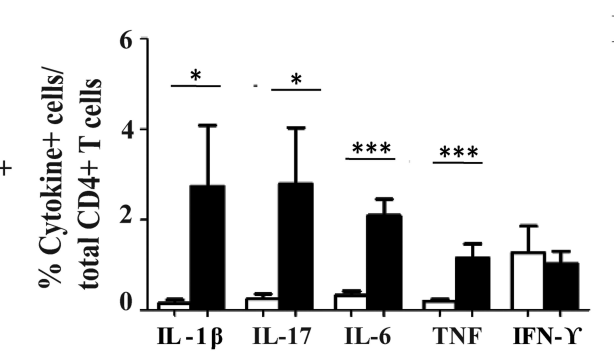

CD4+

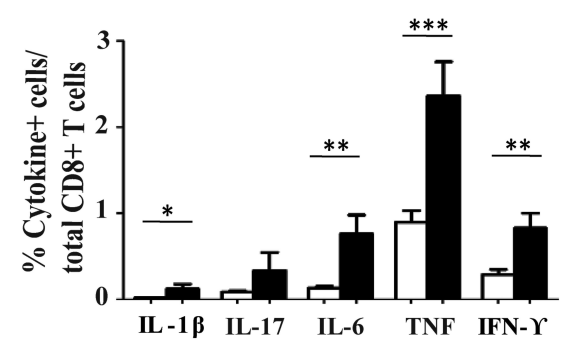

C
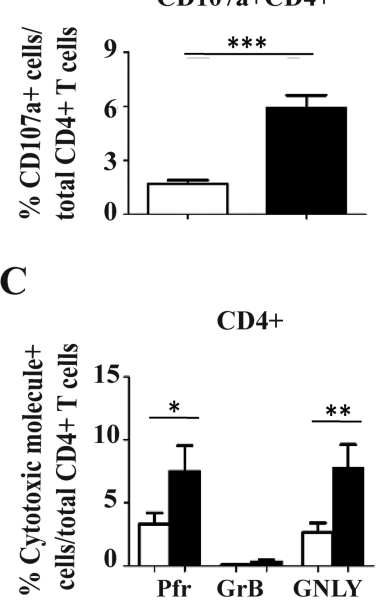

CD107a+CD8+

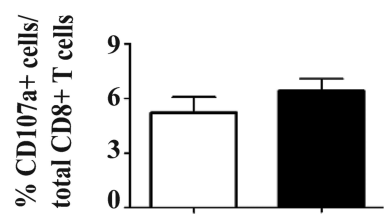

CD8+

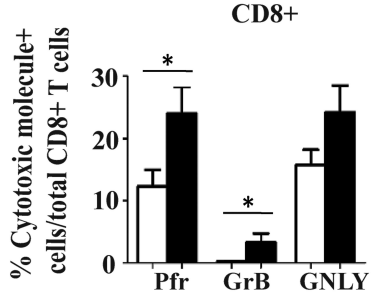

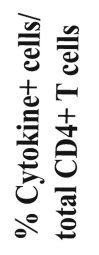

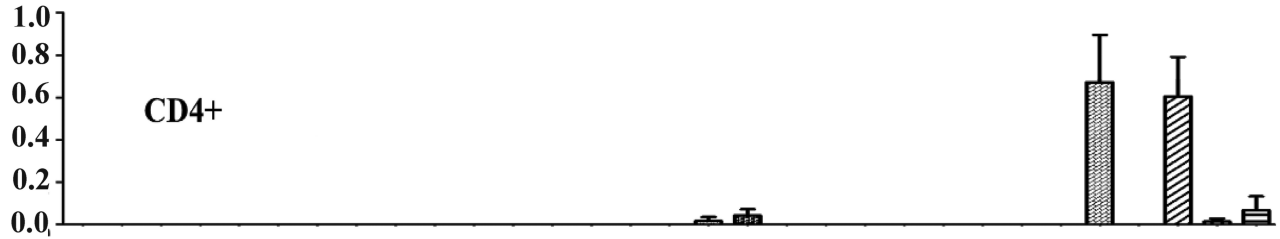

\begin{tabular}{|c|c|c|c|c|c|c|c|c|c|c|c|c|c|c|c|c|c|c|c|c|c|c|}
\hline IL-1及 & + & & + & + & + & & & & & ++ & + & + & + & + & + & + & ++ & & & & & + \\
\hline IL-17 & $+\mid$ & $+\quad+$ & + & & + & + & + & + & & + & + & & & + & & & + & & + & + & + & + \\
\hline IL-6 & ++ & + & & + & + & + & + & & + & & & + & + & + & & + & & + & + & + & & + \\
\hline TNF & $+\mid+$ & + & + & + & & + & & + & + & + & & + & & & & & + & & + & & + & + \\
\hline IFNY & $+\square$ & + & + & + & + & & + & + & + & + & $+\quad+$ & & + & & + & & & + & + & & + & \\
\hline
\end{tabular}

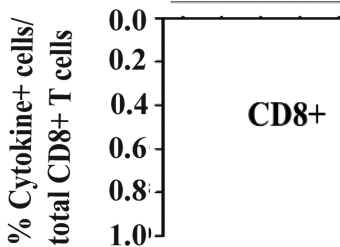

Figure 2. Ex vivo T cell cytokine and cytotoxic molecule production in IPFP of patients with knee OA compared with patient-matched peripheral blood. T cells were isolated from peripheral blood and IPFP of patients with knee OA. Intracellular cytokine staining and degranulation assay was performed to evaluate the levels of T cells producing IL-1 $\beta$, IL-17, IL-6, TNF, IFN- $\gamma$, perforin, granzyme B, and granulysin. T cells were grouped based on their coreceptor expression of CD4 or CD8. Because of the limited number of cells isolated from IPFP, only ex vivo conditions were used to determine the levels of cytokine-producing and cytotoxic molecule-producing T cells. Not all patients were matched between both tissues. Shown are mean values. Error bars represent SD values. Peripheral blood (white bars), $\mathrm{n}=26$; IPFP (black bars), $\mathrm{n}=17$. * $\mathrm{p} \leq 0.05$, ** $\mathrm{p} \leq 0.01$, *** $\mathrm{p} \leq 0.001$. Prf: perforin; GrB: granzyme B; GNLY: granulysin; IL: interleukin; TNF: tumor necrosis factor; OA: osteoarthritis; IFN: interferon; PBMC: peripheral blood mononuclear cells; IPFP: infrapatellar fat pads.

clones and those that were not predominant (present in 2 or more patients; Figure 4B, Table 2). In IPFP, shared TRBV usage included TRBV 14, 15, 27, and 29-1 (Figure 4B). With the number of clones that we obtained, the data show that activated CD8+ T cells in IPFP differ from those in peripheral blood of the same individual. Also, the shared TRBV usages observed in activated CD8+ IPFP T cells supports the notion of a common driver among patients with knee OA in specific
$\mathrm{CD} 8+\mathrm{T}$ cell recruitment or proliferation. These drivers would more likely be stimuli local to the joint because peripheral blood-activated CD8+ T cells do not reflect the repertoire hierarchy in IPFP.

\section{DISCUSSION}

$\mathrm{T}$ cells are effector cells of the adaptive immune response that function by proliferation, cytokine production, cytotox-

Personal non-commercial use only. The Journal of Rheumatology Copyright (C) 2019. All rights reserved. 
A

IPFP
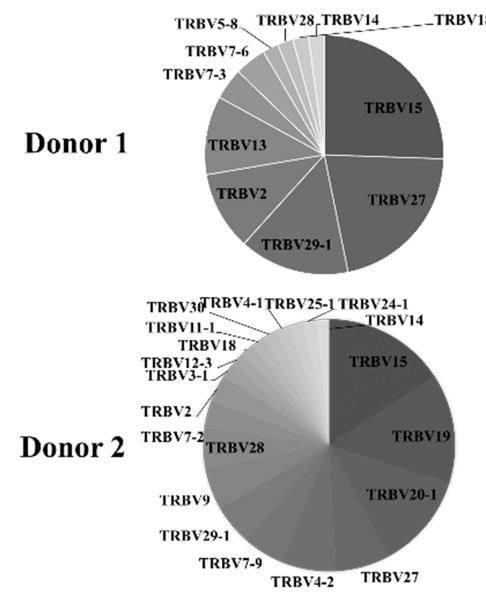

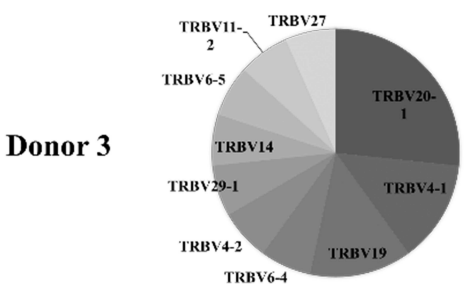

PB
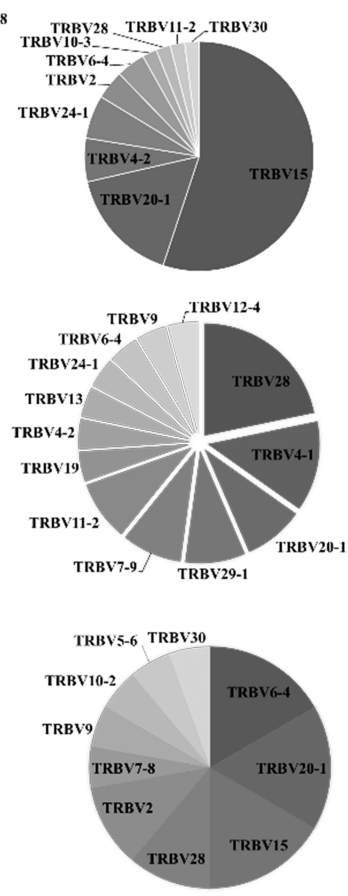

B
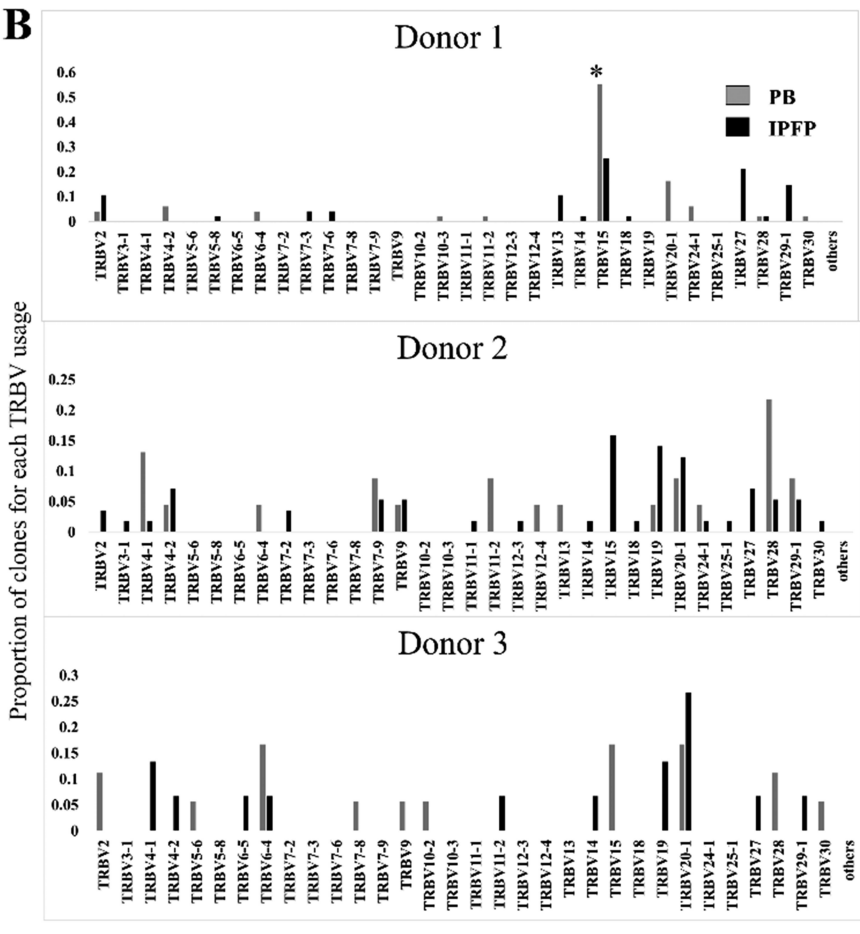

Figure 3. TRBV usage of activated (CD69+) CD8+ T cells in peripheral blood and IPFP of 3 patients with knee OA. Number of clones: Donor 1, PB $\mathrm{n}=23$, IPFP $n=59$; Donor 2, PB $n=49$, IPFP $n=47$; Donor 3, PB $n=21$, IPFP $n=16$. A. Proportions of each TRBV usage in IPFP and peripheral blood of donors 1,2, and 3. B. Shared TRBV usage by activated CD8+ T cells in peripheral blood and IPFP in each donor. * This TRBV15 usage by Donor 1 contained clones that were identical (same CDR3- $\beta$ ) in 25/27 clones. X axis represents each TRBV. Y axis represent the proportion of clones for each TRBV usage. OA: osteoarthritis; PB: peripheral blood; IPFP: infrapatellar fat pads; TRBV: T cell receptor $\beta$-variable gene chain.

Table 2. Predominant TRBV usage of activated (CD69+) CD8+ T cells from patients with knee OA, from peripheral blood and IPFP.

\begin{tabular}{lcc}
\hline Donor \# & PB & IPFP \\
\hline 1 & TRBV15 $(55)^{*}$ & TRBV15 (26) \\
& TRBV20-1 (17) & TRBV27 (21) \\
& TRBV4-2 (6)** & TRBV29-1 (15) \\
& TRBV24-1 (6)** & TRBV2 (11) \\
& & TRBV13 (11) \\
2 & TRBV28 (22) & TRBV15 (16) \\
& TRBV4-1 (13) & TRBV19 (14) \\
3 & TRBV20-1 (9)*** & TRBV20-1 (27) \\
& TRBV6-4 (17) & TRBV4-1 (13) \\
& TRBV20-1 (17) & TRBV19 (13) \\
& TRBV15 (17) & TRBV29-1 (7)***
\end{tabular}

Data in parentheses are percentages. Activated CD8+ T cells from peripheral blood (PB) and IPFP of 3 knee OA patients were analyzed for the TCR repertoire by determining their TRBV usage. Shown in this table are the predominant TRBV usages (> 10\%) of each donor and from each tissue. Percentages represent the proportion of each TRBV usage from all clones. Number of clones: Donor 1, PB $n=23$, IPFP $n=59$; Donor 2, PB $n=49$, IPFP $n=47$; Donor $3, P B n=21$, IPFP $n=16$. *This TRBV15 usage by Donor 1 contained clones that were identical (same CDR3 $\beta$ ) in 25/27 clones. ** TRBV usage of subsequent hierarchy in PB of Donor 1 was included to compensate for the biased large proportion of TRBV15 usage. $* * *$ Clones that were $<10 \%$ in proportion included to illustrate the overall view of TRBV usage among donors. Values in bold face represent TRBV usages that are overlapping among donors. TRBV: T cell receptor $\beta$-variable gene chain; OA: osteoarthritis; IPFP: infrapatellar fat pads; TCR: T cell receptor. 
Donor 3

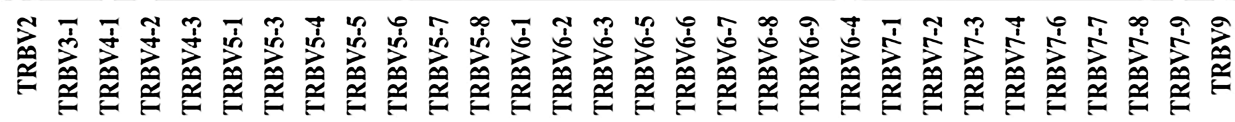
\begin{tabular}{|r}
30 \\
25 \\
20 \\
15 \\
10 \\
5 \\
0
\end{tabular}

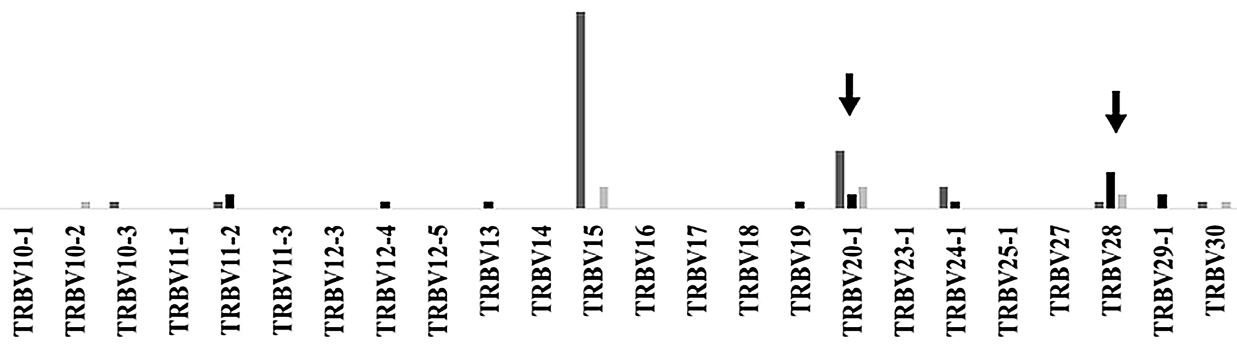

B

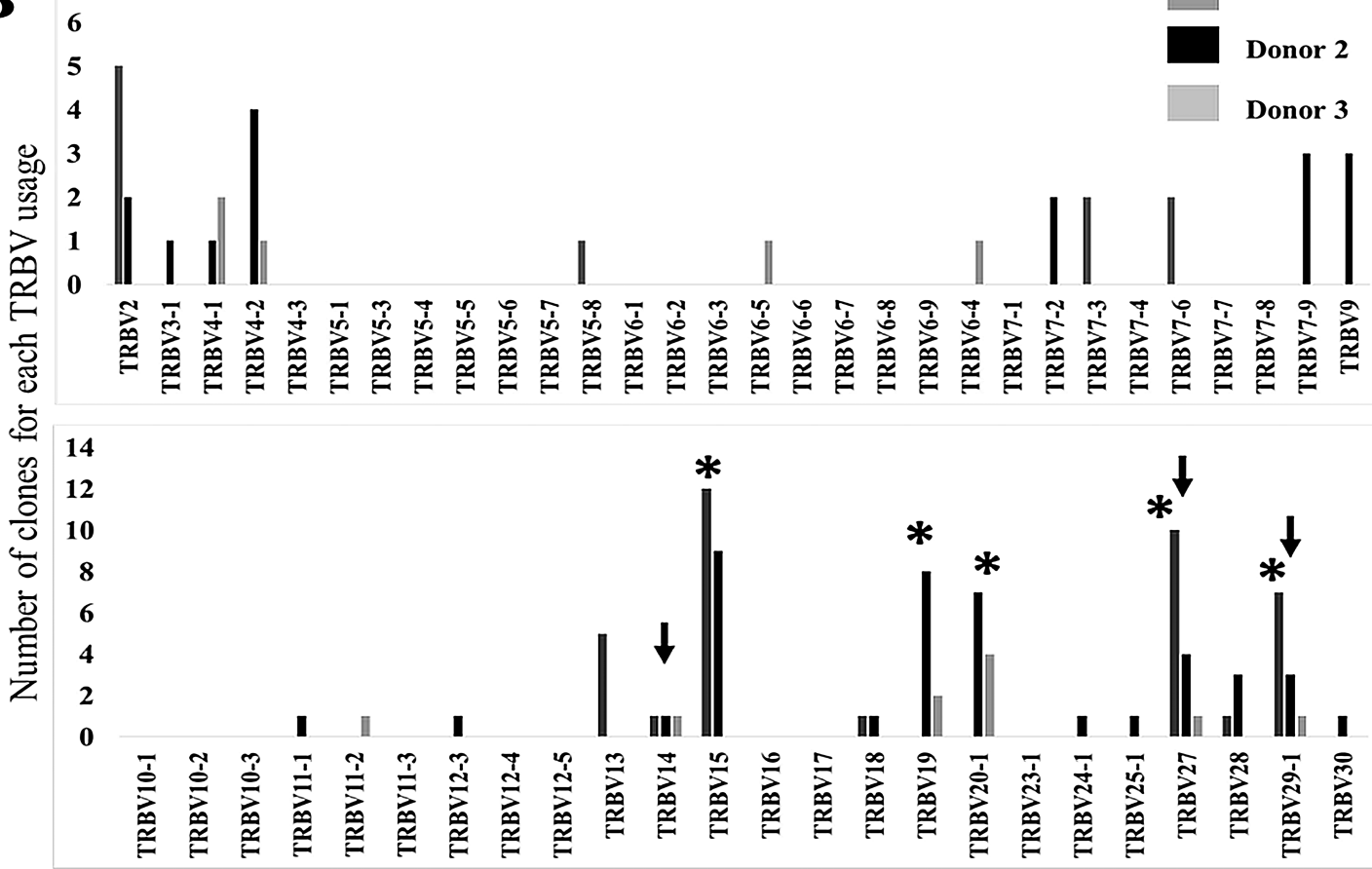

Figure 4. Common shared TRBV usage among 3 patients with knee OA in activated CD8+ T cells of peripheral blood and IPFP. A TRBV usage of peripheral blood activated CD8+ T cells from 3 patients with knee OA. Black arrows represent TRBV chains that are shared among all 3 donors. B. TRBV usage of IPFP-activated CD8+ T cells from 3 patients. Black arrows represent TRBV chains that are shared among all 3 donors. Asterisks $(*)$ represent TRBV that were selected for their dominant presence in IPFP. TRBV: T cell receptor $\beta$-variable gene chain; OA: osteoarthritis; IPFP: infrapatellar fat pads; PBMC: peripheral blood mononuclear cells.

icity, and differentiation ${ }^{44}$ and have been described in knee OA because of their presence in synovial fluids, synovium linings, and IPFP ${ }^{10,13,25}$. Our study focused on understanding the role of T cells in IPFP and peripheral blood of patients with knee OA by comparing their phenotypes to healthy individuals. In addition, we also compared the TCR reper- 
toire of IPFP and peripheral blood $\mathrm{T}$ cells in 3 patients with knee OA.

Here, we show that in peripheral blood of patients with knee OA, there was a tendency of CD4+ and CD8+ T cells to have lower cytokine production and an increase in cytotoxic molecule production. Despite previous studies showing that increased frequency of $\mathrm{CD} 8+\mathrm{T}$ cells is related with knee OA with increasing age, our data suggest that $\mathrm{T}$ cells driving inflammation in knee OA may also have altered function in addition to disproportionate CD4+/CD8+ imbalances. Aging causes a decline in immune function referred to as immunosenescence ${ }^{45}$ and is accompanied by low-grade inflammation of the host ${ }^{46}$. Because of differences between healthy individuals and patients with knee OA in our study, interpretation of results should take this factor into account. T cells are most severely affected and have increased $\mathrm{CD} 8+\mathrm{T}$ cell clonality because of the decline in CD4+ T cell survival, presence of chronic simulation by latent viral infection, thymic involution, and accumulation of immune exposure history ${ }^{45}$. In other words, aging, along with additional driving forces, may polarize such CD4+ T cells to a declining state with dysfunctional phenotypes in knee OA.

Immune function profiling of IPFP $T$ cells suggests their capability in producing proinflammatory cytokines (IL-1 $\beta$, IL-6, TNF), IL-17, and IFN- $\gamma$. Whether this cytokine production from $\mathrm{T}$ cells is increased in IPFP of knee OA is still inconclusive owing to our lack of healthy IPFP from living individuals and postmortem samples. However, it does suggest that the local microenvironment plays a role in driving CD4+ and CD8+ T cells to secrete these cytokines, especially IL-1 $\beta$ and IL-6, whose secretions are tightly regulated ${ }^{32,33,47,48}$. A scenario in which $\mathrm{T}$ cells do secrete IL-6 is present in patients with human T cell lymphotrophic virus infection ${ }^{48}$. However, it is unknown whether certain viral infections are related directly to knee OA, although there are some studies relating viral infections of osteoblasts found in patients with $\mathrm{OA}^{49}$. Regardless, the extent to which IPFP $\mathrm{T}$ cells are contributing to knee inflammation, and whether there are antigen-specific $\mathrm{T}$ cells within the joints, need further investigation. If $\mathrm{T}$ cells are indeed more stringent with their response to inflammatory microenvironment, then $\mathrm{T}$ cells may serve as potential indicators of inflammation in knee OA.

$\mathrm{CD} 8+\mathrm{T}$ cells are the first immune cells to migrate to adipose tissue in response to increased weight and also function as recruiters of macrophages ${ }^{41}$. Thus, it was important to investigate the TCR repertoire of IPFP CD8+ T cells. In support of oligoclonal $\mathrm{T}$ cell analysis previously reported in the synovium ${ }^{50}$, our clonal analysis from 3 patients with knee OA revealed that activated CD8+ T cells within IPFP carry a much narrower repertoire than activated CD8+ $\mathrm{T}$ cells in peripheral blood. In the peripheral blood of 1 patient, there was a very predominant usage of TRBV15. It was also interesting to observe that TRBV15 usage was present in high proportions among IPFP activated CD8+ T cells in at least 2 of the 3 donors tested. In addition, we also observed common TRBV chains that were shared among the 3 IPFP activated $\mathrm{CD} 8+\mathrm{T}$ cell repertoire. Age, being a risk factor for knee OA, may also indirectly influence the TCR repertoire in patients with knee OA because chronic infection in elderly patients is a key factor driving the $\mathrm{T}$ cell landscape to bias toward selected clones ${ }^{45}$. An increase in weight may also contribute to this phenomenon; in adipose tissues of obese mice, limited TCR diversity is observed ${ }^{42}$. However, the predominant $\beta$-chain usage in peripheral blood differed from that of IPFP $T$ cells, suggesting a selective pressure for CD8+ T cells that are accumulated in the IPFP. Regardless, more studies are required to distinguish whether the selected clones present in IPFP are a result of migration of selected clones or local clonal selection and expansion that occurs within the IPFP.

We show here that $\mathrm{T}$ cells are capable of producing soluble mediators locally and are biased toward cytotoxicity. Also, activated CD8+ T cells within the IPFP contained a limited repertoire diversity when compared to peripheral blood $\mathrm{T}$ cells (Supplementary Figure 4, available with the online version of this article). Unknown forces driving the selection, recruitment, and altered function of $\mathrm{T}$ cells within the knee joint need to be further studied.

\section{ACKNOWLEDGMENT}

We thank the participating patients and clinical staff members at the orthopedics ward for providing and collecting samples that were used in this study.

\section{ONLINE SUPPLEMENT}

Supplementary material accompanies the online version of this article.

\section{REFERENCES}

1. Loeser RF, Goldring SR, Scanzello CR, Goldring MB Osteoarthritis: a disease of the joint as an organ. Arthritis Rheum 2012;64:1697-707.

2. Neogi T, Zhang Y. Epidemiology of osteoarthritis. Rheum Dis Clin North Am 2013;39:1-19.

3. Berenbaum F. Osteoarthritis as an inflammatory disease (osteoarthritis is not osteoarthrosis!). Osteoarthritis Cartilage 2013;21:16-21

4. Kapoor M, Martel-Pelletier J, Lajeunesse D, Pelletier JP, Fahmi H. Role of proinflammatory cytokines in the pathophysiology of osteoarthritis. Nat Rev Rheumatol. 2011;7:33-42.

5. Scanzello CR. Role of low-grade inflammation in osteoarthritis. Curr Opin Rheumatol 2017;29:79-85.

6. Liu-Bryan R, Terkeltaub R. Emerging regulators of the inflammatory process in osteoarthritis. Nat Rev Rheumatol 2015;11:35-44.

7. Orlowsky EW, Kraus VB. The role of innate immunity in osteoarthritis: when our first line of defense goes on the offensive. J Rheumatol 2015;42:363-71.

8. Liu-Bryan R. Synovium and the innate inflammatory network in osteoarthritis progression. Curr Rheumatol Rep 2013;15:323.

9. Haseeb A, Haqqi TM. Immunopathogenesis of osteoarthritis. Clin Immunol 2013;146:185-96.

10. Sakkas LI, Platsoucas CD. The role of T cells in the pathogenesis of osteoarthritis. Arthritis Rheum 2007;56:409-24.

11. Robinson WH, Lepus CM, Wang Q, Raghu H, Mao R, Lindstrom $\mathrm{TM}$, et al. Low-grade inflammation as a key mediator of the

Personal non-commercial use only. The Journal of Rheumatology Copyright @ 2019. All rights reserved. 
pathogenesis of osteoarthritis. Nat Rev Rheumatol 2016;12:580-92.

12. Sellam J, Berenbaum F. The role of synovitis in pathophysiology and clinical symptoms of osteoarthritis. Nat Rev Rheumatol 2010;6:625-35.

13. Ioan-Facsinay A, Kloppenburg M. An emerging player in knee osteoarthritis: the infrapatellar fat pad. Arthritis Res Ther 2013;15:225.

14. Eymard F, Chevalier X. Inflammation of the infrapatellar fat pad. Joint Bone Spine 2016;83:389-93.

15. Apinun J, Sengprasert P, Yuktanandana P, Ngarmukos S, Tanavalee A, Reantragoon R. Immune mediators in osteoarthritis: infrapatellar fat pad-infiltrating CD8+ T cells are increased in osteoarthritic patients with higher clinical radiographic grading. Int J Rheumatol 2016;2016:9525724.

16. Favero M, El-Hadi H, Belluzzi E, Granzotto M, Porzionato A, Sarasin G, et al. Infrapatellar fat pad features in osteoarthritis: a histopathological and molecular study. Rheumatology 2017;56:1784-93

17. Kontny E, Plebanczyk M, Lisowska B, Olszewska M, Maldyk P, Maslinski W. Comparison of rheumatoid articular adipose and synovial tissue reactivity to proinflammatory stimuli: contribution to adipocytokine network. Ann Rheum Dis 2012;71:262-7.

18. Belluzzi E, El Hadi H, Granzotto M, Rossato M, Ramonda R, Macchi V, et al. Systemic and local adipose tissue in knee osteoarthritis. J Cell Physiol 2017;232:1971-8.

19. Chuckpaiwong B, Charles HC, Kraus VB, Guilak F, Nunley JA. Age-associated increases in the size of the infrapatellar fat pad in knee osteoarthritis as measured by 3 T MRI. J Orthop Res 2010;28:1149-54.

20. Pan F, Han W, Wang X, Liu Z, Jin X, Antony B, et al. A longitudinal study of the association between infrapatellar fat pad maximal area and changes in knee symptoms and structure in older adults. Ann Rheum Dis 2015;74:1818-24.

21. Han W, Cai S, Liu Z, Jin X, Wang X, Antony B, et al. Infrapatellar fat pad in the knee: is local fat good or bad for knee osteoarthritis? Arthritis Res Ther 2014;16:R145.

22. Han W, Aitken D, Zhu Z, Halliday A, Wang X, Antony B, et al. Signal intensity alteration in the infrapatellar fat pad at baseline for the prediction of knee symptoms and structure in older adults: a cohort study. Ann Rheum Dis 2016;75:1783-8.

23. Cai J, Xu J, Wang K, Zheng S, He F, Huan S, et al. Association between infrapatellar fat pad volume and knee structural changes in patients with knee osteoarthritis. J Rheumatol 2015;42:1878-84.

24. Klein-Wieringa IR, de Lange-Brokaar BJ, Yusuf E, Andersen SN, Kwekkeboom JC, Kroon HM, et al. Inflammatory cells in patients with endstage knee osteoarthritis: a comparison between the synovium and the infrapatellar fat pad. J Rheumatol 2016;43:771-8.

25. Klein-Wieringa IR, Kloppenburg M, Bastiaansen-Jenniskens YM, Yusuf E, Kwekkeboom JC, El-Bannoudi H, et al. The infrapatellar fat pad of patients with osteoarthritis has an inflammatory phenotype. Ann Rheum Dis 2011;70:851-7.

26. Hsieh JL, Shiau AL, Lee CH, Yang SJ, Lee BO, Jou IM, et al. CD8+ $\mathrm{T}$ cell-induced expression of tissue inhibitor of metalloproteinses-1 exacerbated osteoarthritis. Int J Mol Sci 2013;14:19951-70.

27. Shen PC, Wu CL, Jou IM, Lee CH, Juan HY, Lee PJ, et al. T helper cells promote disease progression of osteoarthritis by inducing macrophage inflammatory protein-1gamma. Osteoarthritis Cartilage 2011;19:728-36.

28. Wang GC, Dash P, McCullers JA, Doherty PC, Thomas PG. T cell receptor $\alpha \beta$ diversity inversely correlates with pathogen-specific antibody levels in human cytomegalovirus infection. Sci Transl Med 2012;4:128ra42.

29. Goldring MB. Osteoarthritis and cartilage: the role of cytokines. Curr Rheumatol Rep 2000;2:459-65.

30. Gabay C. Interleukin-6 and chronic inflammation. Arthritis Res
Ther 2006;8 Suppl 2:S3.

31. Sokolove J, Lepus CM. Role of inflammation in the pathogenesis of osteoarthritis: latest findings and interpretations. Ther Adv Musculoskelet Dis 2013;5:77-94.

32. Li T, He S. Induction of IL-6 release from human T cells by PAR-1 and PAR-2 agonists. Immunol Cell Biol 2006;84:461-6.

33. Martin BN, Wang C, Zhang CJ, Kang Z, Gulen MF, Zepp JA, et al. $\mathrm{T}$ cell-intrinsic ASC critically promotes $\mathrm{T}(\mathrm{H}) 17$-mediated experimental autoimmune encephalomyelitis. Nat Immunol 2016;17:583-92.

34. Lubberts E. The IL-23-IL-17 axis in inflammatory arthritis. Nat Rev Rheumatol 2015; 11:562.

35. Schoenborn JR, Wilson CB. Regulation of interferon-gamma during innate and adaptive immune responses. Adv Immunol 2007; 96:41-101.

36. Manek NJ, Hart D, Spector TD, MacGregor AJ. The association of body mass index and osteoarthritis of the knee joint: an examination of genetic and environmental influences. Arthritis Rheum 2003;48:1024-9.

37. Yusuf E, Nelissen RG, Ioan-Facsinay A, Stojanovic-Susulic V, DeGroot J, van Osch G, et al. Association between weight or body mass index and hand osteoarthritis: a systematic review. Ann Rheum Dis 2010;69:761-5.

38. Jiang L, Xie X, Wang Y, Wang Y, Lu Y, Tian T, et al. Body mass index and hand osteoarthritis susceptibility: an updated meta-analysis. Int J Rheum Dis 2016;19:1244-54.

39. Courties A, Sellam J, Berenbaum F. Metabolic syndrome-associated osteoarthritis. Curr Opin Rheumatol 2017;29:214-22.

40. Santangelo KS, Radakovich LB, Fouts J, Foster MT. Pathophysiology of obesity on knee joint homeostasis: contributions of the infrapatellar fat pad. Horm Mol Biol Clin Investig 2016;26:97-108.

41. Nishimura S, Manabe I, Nagasaki M, Eto K, Yamashita H, Ohsugi $\mathrm{M}$, et al. CD8+ effector T cells contribute to macrophage recruitment and adipose tissue inflammation in obesity. Nat Med 2009;15:914-20.

42. Yang H, Youm YH, Vandanmagsar B, Ravussin A, Gimble JM, Greenway $\mathrm{F}$, et al. Obesity increases the production of proinflammatory mediators from adipose tissue $\mathrm{T}$ cells and compromises TCR repertoire diversity: implications for systemic inflammation and insulin resistance. J Immunol 2010;185:1836-45.

43. Ponchel F, Burska AN, Hensor EM, Raja R, Campbell M, Emery P, et al. Changes in peripheral blood immune cell composition in osteoarthritis. Osteoarthritis Cartilage 2015;23:1870-8.

44. Masopust D, Schenkel JM. The integration of T cell migration, differentiation and function. Nat Rev Immunol 2013;13:309-20.

45. Fulop T, Larbi A, Pawelec G. Human T cell aging and the impact of persistent viral infections. Front Immunol 2013;4:271.

46. Franceschi C, Bonafe M, Valensin S. Human immunosenescence: the prevailing of innate immunity, the failing of clonotypic immunity, and the filling of immunological space. Vaccine 2000;18:1717-20.

47. Ming JE, Granelli-Piperno A. Distinctive features in the production of IL-6 by human T cells. Cell Immunol 1990;130:437-45.

48. Villiger PM, Cronin MT, Amenomori T, Wachsman W, Lotz M. IL-6 production by human $\mathrm{T}$ lymphocytes. Expression in HTLV-1-infected but not in normal T cells. J Immunol 1991;146:550-9.

49. Chen W, Foo SS, Rulli NE, Taylor A, Sheng KC, Herrero LJ, et al. Arthritogenic alphaviral infection perturbs osteoblast function and triggers pathologic bone loss. Proc Natl Acad Sci U S A 2014;111:6040-5.

50. Stamenkovic I, Stegagno M, Wright KA, Krane SM, Amento EP, Colvin RB, et al. T lymphocyte infiltrates in inflammatory synovia are oligoclonal. Transplant Proc 1988;20:315-9.

Personal non-commercial use only. The Journal of Rheumatology Copyright $\subset$ (2019. All rights reserved. 